\title{
Testing the $v_{\max }$ scaling relation
}

\author{
H. R. Coelho ${ }^{1,2, a}$, W. J. Chaplin ${ }^{1,2}$, S. Basu ${ }^{3}$, A. Serenelli ${ }^{4}$, A. Miglio ${ }^{1,2}$, and D. Reese ${ }^{1,2}$
}

1 School of Physics and Astronomy, University of Birmingham, Edgbaston, Birmingham, B15 2TT, UK.

2 Stellar Astrophysics Centre (SAC),Department of Physics and Astronomy, Aarhus University, Ny Munkegade 120, DK-8000 Aarhus C, Denmark.

3 Department of Physics and Astronomy, Yale University, P.O. Box 208101, New Haven, CT, 06520, USA.

4 Instituto de Ciencias del Espacio (CSIC - IEEC), Facultad de Ciencies, Campus UAB, E-08193 Bellaterra, Spain.

\begin{abstract}
Two key global seismic quantities are relevant to estimate the fundamental properties of a star: the frequency of maximum power $\left(v_{\max }\right)$ and the large frequency separation $(\Delta v)$. The focus of this work is to test the $v_{\max }$ scaling relation in order to ascertain it's level of accuracy. Here we report our results using artificial data and real Kepler data, based on a grid-modelling approach.
\end{abstract}

\section{Introduction}

In order to test the $v_{\max }$ scaling relation, artificial data were created to conduct a hare-and-hounds exercise. This exercise consists in using the observable quantities of the artificial stars as input to different grid-modelling pipelines to estimate fundamental stellar properties. By comparing the results obtained by the pipeline with the actual values for the artificial stars, it is possible to see how effective the grid pipelines are. In this work, we created a set of artificial data that mimics the properties of 337 main-sequence and sub-giants stars observed by the Kepler spacecraft [1].

We used three different codes to analyse the data: PARAM [2], Yale-Birmingham [3][4] and BeSPP (Serenelli et al., in preparation). The results obtained for $v_{\max }$ are divided in two categories: one obtained by using Teff, $[\mathrm{Fe} / \mathrm{H}]$, and $\Delta v$ as input in the grid-pipelines, where $\Delta v$ was obtained through the use of scaling relations, and a second set of results obtained in a similar way using $T_{\text {eff }},[\mathrm{Fe} / \mathrm{H}]$ and $\Delta v$ as input, but this time $\Delta v$ is estimated using the individual frequencies of the stars. Using results from the artificial data as a guide, preliminary results for the real Kepler data suggests that there is no significant bias in the $v_{\max }$ scaling relation (Coelho et al., in preparation.).

\section{Artificial data}

The artificial data were created by selecting models from a grid of stellar evolutionary tracks. The grid was built using the MESA code (Modules for Experiments in Stellar Astrophysics [5]). Each model matches the properties of one of the 337 stars observed by the Kepler spacecraft, such as $\Delta v, T_{\text {eff }}$ and $[\mathrm{Fe} / \mathrm{H}]$.

Assuming the temperatures and metallicities to be unbiased, the ratio $\left(v_{\text {data }} / v_{\text {grid }}\right)$, where $v_{\text {data }}$ are the

\footnotetext{
a e-mail: hugorc@ibison.ph.bham.ac.uk
} 

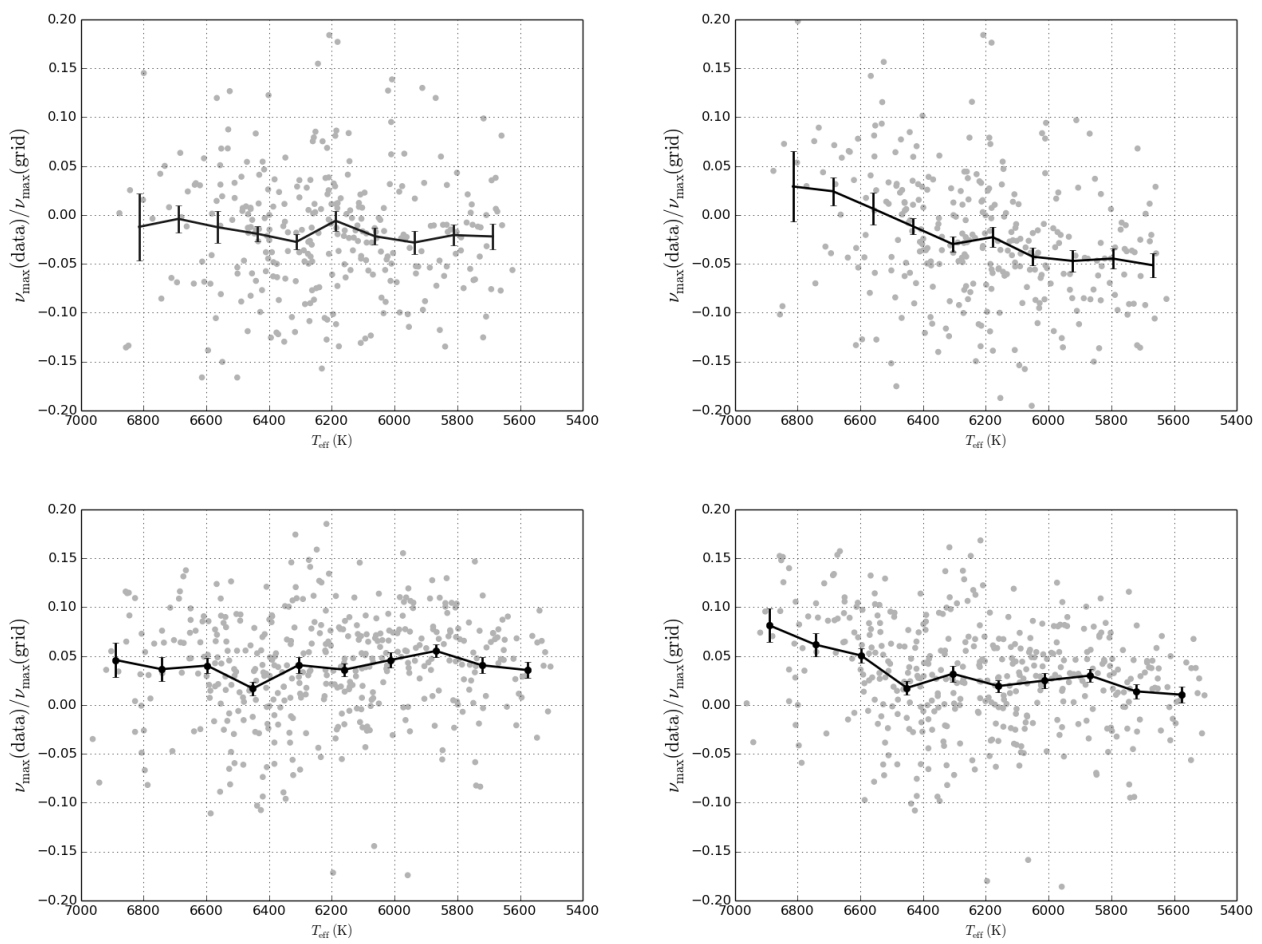

Fig. 1. Top: results obtained by the BeSPP code for the artificial data code when the ratio $v_{\text {data }} / v_{\text {grid }}$ is plotted as function of effective temperature. On the top right, the $\Delta v$ scaling relation was used to calculate the values of the large separation. The effects of the bias in the $\Delta v$ scaling relation will affect the results [6]. On the top left, $\Delta v$ was estimated using individual frequencies. Bottom: same as top, but for the real stars observed by Kepler.

observed values, will provide a direct estimate of the bias in the $v_{\max }$ scaling, i.e., the fractional amount by which $v_{\max }$ is over or underestimated relative to some notional parameter that scales exactly as $g T_{\mathrm{eff}}^{-\frac{1}{2}}$. Figure 1 show results for the artificial data (top) and the real stars (bottom). When individual frequencies are used to obtain $\Delta v$, the effects of the bias in the $\Delta v$ scaling relation [6] are suppressed.

The procedure to obtain $v_{\text {grid }}$ for the real stars is basically the same as used for the analysis of the artificial data. The preliminary results show no significant bias in the $v_{\max }$ scaling relation was found at the level of precision of the data presented here.

\section{References}

1. Chaplin, W. J. et al., ApJS 210:1, (2014) 22

2. da Silva L., et al., A\&A 458, (2006) 609

3. Basu, S., Chaplin, W. J., Elsworth, Y., ApJ 710, (2010) 1596

4. Basu, S., Verner, G. A., Chaplin, W. J., Elsworth, Y., ApJ 746, (2012) 76

5. Paxton, B., Bildstein, L., Dotter, A., et al., ApJS 192, (2011) 3

6. White, T. R., Huber, D., Maestro, V., et al., MNRAS 433, (2013) 1262 